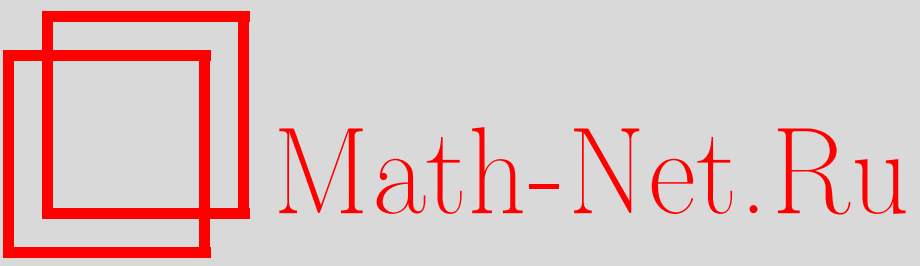

В. М. Бухштабер, Т. Е. Панов, Алгебраическая топология многообразий, определяемых простыми многогранниками, УМH, 1998, том 53, выпуск 3, 195-196

DOI: https://doi.org/10.4213/rm20

Использование Общероссийского математического портала Math-Net.Ru подразумевает, что вы прочитали и согласны с пользовательским соглашением

http://www.mathnet.ru/rus/agreement

Параметры загрузки:

IP: 18.207 .199 .55

26 апреля 2023 г., 14:05:59 


\title{
АЛГЕБРАИЧЕСКАЯ ТОПОЛОГИЯ МНОГООБРАЗИЙ, ОПРЕДЕЛЯЕМЫХ ПРОСТЫМИ МНОГОГРАННИКАМИ
}

\author{
В. М. БУХШТАБЕР, Т. Е. ПАНОВ
}

Выпукльй $n$-мерный многогранник называется $n p$ остылм, если в каждой его вершине сходится ровно $n$ граней коразмерности один. С каждым таким многогранником канонически связывается гладкое многообразие. Основной целю данной работы является исследование связи комбинаторной структуры простых многогранников с топологией этих многообразий.

Пусть $P^{n}$-простой многогранник, $f_{i}$ - число его граней коразмерности $(i+1), 0 \leqslant i \leqslant n-1$ и $\mathscr{F}=\left(F_{1}^{n-1}, \ldots, F_{m}^{n-1}\right)-$ множество его граней коразмерности один, $m=f_{0}$. Фиксируем в качестве основного некоторое коммутативное колцо $k$. Введем кольцо граней $k(P)$ [1].

Для любого простого многогранника $P^{n}$ можно следующим образом построить два топологических пространства $\mathscr{Z}_{P}$ и $B_{T} P\left(\right.$ см. [2]). Задав стандартньй базис $\left\{e_{1}, \ldots, e_{m}\right\}$ в $\mathbb{Z}^{m}$, определим канонические подгруппы $T_{i_{1}}^{k}, \ldots i_{k} \subset T^{m}$ как торы, соответствущие подрешеткам в $\mathbb{Z}^{m}$, натянутым на векторы $e_{i_{1}}, \ldots, e_{i_{k}}$. Тогда определим $\mathscr{Z}_{P}=\left(T^{m} \times P^{n}\right) / \sim$, где $\left(g_{1}, p\right) \sim\left(g_{2}, q\right)$ $\Longleftrightarrow p=q$ и $g_{1} g_{2}^{-1} \in T_{i_{1}}^{k}, \ldots, i_{k}$. Здесь $i_{1}, \ldots, i_{k}$ - набор индексов всех $(n-1)$-граней, содержащих точку $p \in P^{n}$. Заметим, что $\operatorname{dim} \mathscr{Z}_{P}=m+n$. На $\mathscr{Z}_{P}$ действует тор $T^{m}$ с пространством орбит $P^{n}$. Используя это действие, положим $B_{T} P=E T^{m} \times_{T^{m}} \mathscr{Z}_{P}$, где $E T^{m}$ - стягиваемое пространство универсального $T^{m}$-расслоения над $B T^{m}=\left(\mathbb{C} P^{\infty}\right)^{m}$. Ясно, что гомотопический тип пространства $B_{T} P$ определяется простым многогранником $P^{n}$.

Лемма 1. Любой простой многогранник $P^{n}$ имеет естественную структуру кубического комплекса $\mathscr{C}$, составленного из $s=f_{n-1}$ кубов $I_{v}^{n}$ размерности $n$, соответствующих вериинам $v \in P^{n}$. Комплекс $\mathscr{C}$ имеет $1+f_{0}+f_{1}+\cdots+f_{n-1}$ вершин. Определено естественное вложение $i_{P}$ комплекса $\mathscr{C}$ в граничу

Вложение $\mathscr{C} \hookrightarrow I^{m}$ обладает следующим свойством:

$$
\begin{aligned}
& \text { Если } v=F_{i_{1}}^{n-1} \cap \cdots \cap F_{i_{n}}^{n-1} \text {, то куб } I_{v}^{n} \subset P^{n} \text { отображается на } n \text {-мерную грань } \\
& \text { куба } I^{m} \text {, выделяемую } m-n \text { уравнениями } x_{j}=1, j \notin\left\{i_{1}, \ldots, i_{n}\right\} .
\end{aligned}
$$

Рассмотрим теперь стандартный полидиск $\left(D^{2}\right)^{m}=\left\{\left(z_{1}, \ldots, z_{m}\right) \in \mathbb{C}^{m}|| z_{i} \mid \leqslant 1\right\} \subset \mathbb{C}^{m}$. Стандартное диагональное действие тора $T^{m}$ на $\mathbb{C}^{m}$ задает действие $T^{m}$ на $\left(D^{2}\right)^{m}$, пространством орбит которого является $I^{m}$.

ТеОРема 2. Пусть $P^{n}$ - простой многогранник. Пространство $\mathscr{Z}_{P}$ имеет каноническую структуру гладкого многообразия размерности $m+n$, относительно которой действие $T^{m}$ является гладким. Вложение $i_{P}: P^{n} \hookrightarrow I^{m}$, построенное в лемме 1 , индуцирует $T^{m}$-эквивариантное вложение $i_{e}: \mathscr{Z}_{P} \hookrightarrow\left(D^{2}\right)^{m} \subset \mathbb{C}^{m}$ с тривиальным нормальным расслоением.

ДокАЗАТЕЛЬСтво. Пусть $\rho: \mathscr{Z}_{P} \rightarrow P^{n}$ - проекция на пространство орбит. Тогда из конструкции $\mathscr{Z}_{P}$ следует, что для любого куба $I_{v}^{n} \subset \mathscr{Z}_{P}$ (см. лемму 1 ) мы имеем $\rho^{-1}\left(I_{v}^{n}\right)=$ $\left(D^{2}\right)^{n} \times T^{m-n}$, где $\left(D^{2}\right)^{n}$ - полидиск в $\mathbb{C}^{n}$ со стандартным действием тора $T^{n}$. Отсюда вытекает, что $\mathscr{Z}_{P}$ представляется в виде объединения подмножеств $B_{v} \cong\left(D^{2}\right)^{n} \times T^{m-n}$. Тем самым $\mathscr{Z}_{P}$ приобретает структуру гладкого многообразия с гладким действием $T^{m}$. Легко видеть, что вложение в куб $I^{m}$ грани $I^{n}$, заданной $m-n$ уравнениями вида $x_{j}=1$, индуцирует эквивариантное вложение пространства вида $\left(D^{2}\right)^{n} \times T^{m-n}$ в $\left(D^{2}\right)^{m}$. Тогда утверждения второй части теоремы следуют из свойства (1) вложения $i_{P}: P^{n} \hookrightarrow I^{m}$.

Введем в $B T^{m}=\left(\mathbb{C} P^{\infty}\right)^{m}$ стандартную клеточную структуру, в которой каждое $\mathbb{C} P^{\infty}$ имеет по одной клетке в каждой четной размерности. Далее все когомологии рассматриваются с коэффициентами в основном колшце $k$.

Работа выполнена авторами при частичной финансовой поддержке Российского фонда фундаментальных исследований (грант № 96-01-01404). 
Теорема 3. Пространство $B_{T} P$ может быть реализовано как клеточный подкомплекс в $B T^{m}$, представляющий собой обвединение клеточных подкомплексов $B T_{i_{1}, \ldots, i_{k}}^{k}$ по всем симплексам $\Delta=\left(i_{1}, \ldots, i_{k}\right)$ симплициального комплекса $K_{P}$. При этом вложение $i: B_{T} P \hookrightarrow B T^{m}$ индуцирует эпиморфизм $H^{*}\left(B T^{m}\right)=k\left[v_{1}, \ldots, v_{m}\right] \rightarrow k(P)=H^{*}\left(B_{T} P\right)$.

Простой многогранник $P^{n}$ с $m$ гранями коразмерности один называется $q$-смежностным, если $(q-1)$-остов двойственного симплициального комплекса $K_{P}^{n-1}$ совпадает с $(q-1)$-остовом $(m-1)$-мерного симплекса.

TeOpema 4. 1) $\pi_{1}\left(\mathscr{Z}_{P}\right)=\pi_{1}\left(B_{T} P\right)=0 ;$ 2) $\pi_{2}\left(\mathscr{Z}_{P}\right)=0, \pi_{2}\left(B_{T} P\right)=\mathbb{Z}^{m} ;$ 3) $\pi_{q}\left(\mathscr{Z}_{P}\right)=\pi_{q}\left(B_{T} P\right)$ при $q \geqslant 3$; 4) если $P^{n}$ является $q$-смехностным, то $\pi_{i}\left(\mathscr{Z}_{P}\right)=0$ при $i<2 q+1$ и $\pi_{2 q+1}-$ свободная абелева группа, образующие которой соответствуют мономам $v_{i_{1}} \cdots v_{i_{q+1}} \in I$.

С помощью спектральной последовательности Эйленберга-Мура [3] и теоремы 3 вьводится

ТеОРема 5. Если $k$ - поле, то имеет место изоморфизм алгебр:

$$
H^{*}\left(\mathscr{Z}_{P}\right) \cong \operatorname{Tor}_{k\left[v_{1}, \ldots, v_{m}\right]}(k(P), k) .
$$

В частности, $H^{r}\left(\mathscr{Z}_{P}\right)=\sum_{j-i=r} \operatorname{Tor}_{k\left[v_{1}, \ldots, v_{m}\right]}^{-i, j}(k(P), k), i, j \geqslant 0$.

Используя комплекс Кошуля в качестве резольвенты (см. [4]) и теорему 5, получаем:

Теорема 6. Спектральная последовательность Лере-Серра расслоения $\mathscr{Z}_{P} \times$ $E T^{m} \rightarrow B_{T} P$ со слоем $T^{m}$ вырождается в члене $E_{3}$. Таким образом, имеет место изоморфизм алгебр $H^{*}\left(\mathscr{Z}_{P}\right) \cong H\left[k(P) \otimes \Lambda\left[u_{1}, \ldots, u_{m}\right], d\right], d\left(a \otimes u_{i}\right)=a v_{i} \otimes 1, d(a \otimes 1)=0$, $\operatorname{bideg} a=(0, \operatorname{deg} a), \operatorname{bideg} u_{i}=(-1,2)$, zде $a \in k(P)=k\left[v_{1}, \ldots, v_{n}\right] / I \quad u \quad \Lambda\left[u_{1}, \ldots, u_{m}\right]-$ внешняя алгебра.

СледствиЕ 7. В терминах комплекса $k(P) \otimes \Lambda\left[u_{1}, \ldots, u_{m}\right]$ имеем:

1) представителями фундаментального коцикла многообразия $\mathscr{Z}_{P}$ являются все элементы вида $v_{i_{1}} \cdots v_{i_{n}} \otimes u_{j_{1}} \cdots u_{j_{m-n}}, j_{1}<\cdots<j_{m-n}$, где $\left(i_{1}, \ldots, i_{n}\right)$ - набор индексов граней коразмерности один, сходящихся в некоторой вершине, и $\left\{i_{1}, \ldots, i_{n}, j_{1}, \ldots, j_{m-n}\right\}=\{1, \ldots, m\}$

2) если два әлемента $v_{i_{1}} \cdots v_{i_{p}} \otimes u_{j_{1}} \cdots u_{j_{r}}$ u $v_{k_{1}} \cdots v_{k_{s}} \otimes u_{l_{1}} \cdots u_{l_{t}}$ являются коциклами, то они представляют классы когомологий, двойственные по Пуанкаре, тогда и только тогда, когда $p+s=n, r+t=m-n,\left(i_{1}, \ldots, i_{p}, k_{1}, \ldots, k_{s}\right)$ - набор индексов граней коразмерности один, сходящихся в некоторой вершине, $u\left\{i_{1}, \ldots, i_{p}, j_{1}, \ldots, j_{r}, k_{1}, \ldots, k_{s}\right.$, $\left.l_{1}, \ldots, l_{t}\right\}=\{1, \ldots, m\}$.

Из теоремы 6 при помощи теоремы Гильберта о сизигиях можно получить выражение ряда Пуанкаре колњца $k\left(P^{n}\right)$ в терминах $H^{*}\left(\mathscr{Z}_{P}\right)$. Сопоставляя это выражение с формулой $F(k(P), \lambda)=1+\sum_{i=0}^{n-1} f_{i} \delta^{i+1}, \delta=\lambda^{2} /\left(1-\lambda^{2}\right)$, полученной из комбинаторных соображений (см. [1]), и используя следствие 7 , можно получить комбинаторные соотношения Дена-Соммервилля [5] для простых многогранников.

В заключение отметим, что изложенньй здесь подход к исследованию многообразий $\mathscr{Z}_{P}$ частично сформировался в ходе вьполнения работы [6], и в связи с этим авторы вьражают благодарность Найджелу Рэю.

\section{СПИСОК ЛИТЕРАТУРЫ}

[1] Stanley R. Combinatorics and Commutative Algebra. Boston: Birkhäuser, 1983. [2] Davis M., Januszkiewicz T. // Duke Math. J. 1991. V. 62. № 2. P. 417-451. [3] Smith L. // Trans. Amer. Math. Soc. 1967. V. 129. Р. 58-93. [4] Маклейн С. Гомология. М.: Мир, 1966. [5] Брёнстед А. Введение в теорию выпуклых многогранников. М.: Мир, 1988. [6] Бухштабер В. М., Рэй Н. // УМН. 1998. Т. 53. №2. С. 139-140.

Московский государственный

Принято редколлегией университет им. М.В. Ломоносова

11.05.1998 